(33.33\%) were in junctional rhythm, however, five cases $(55.56 \%)$ were in NSR, three cases (33.33\%) were in AF and one $(11.11 \%)$ was in junctional rhythm by far. Of three cases more than one year after operation, two cases (66.67\%) were in NSR, one case (33.33\%) was in AF. The cardiac function (NYHA) of $84.62 \%$ was gradeI and $15.38 \%$ gradeII. No thrombo-embolism occurred. Sinus bradycardia occurred in two cases during hospitalisation. 13 patients were followed up, reviewing ECG and echocardiography. The overall results of follow-up of cardiac rhythm were NSR in eight cases (61.54\%), AF in four cases $(30.77 \%)$, and junctional rhythm in one case $(7.69 \%)$. Within seven cases whose heart rhythm immediately were able to turn NSR, one case converted to AF later, the rate of maintenance of NSR was $85.71 \%$, however, six patients could not be transferred into NSR instantly, only two cases $(33.33 \%)$ turned into NSR. In NSR group left atrial diameter ECG (LAD) values reduced significantly after cardioversion $(t=6.9580, p=0.0000)$, while LAD in AF group had no significant changes $(t=0.7308, p=0.4925)$. The successful transfer rate was $71.43 \%$ in patients whose AF duration was less than 5 years and was $50 \%$ in patients whose AF duration was more than 5 years. There was no significant difference between the two AF duration groups $\left(X^{2}=0.174, p=0.6788\right)$.

Conclusion Electrical cardioversion after bipolar radiofrequency maze operation was an effective option for the treatment of AF. The best time for cardioversion was within 6 months, which had high immediate success rate and be able to maintain a higher rate of NSR. Those who can be instantly converted to NSR, NSR maintenance rates are relatively high, but cannot be converted to NSR immediately who were less likely to NSR. With the prolonged time, successful rate of turning to NSR after electrical cardioversion reduced gradually. The effect of electrical cardioversion would be better if LAD were significantly reduced.

\section{Related Subjects: Pulmonary Hypertension e0664 CLINICAL RESEARCH ON INFLUENCE FACTORS OF PULMONARY ARTERY SYSTOLIC PRESSURE DERIVED FROM TRICUSPID REGURGITATION BY ECHOCARDIGRAPH}

doi:10.1136/hrt.2010.208967.664

Kang Lihui, Zhang Baowei, Huo Yong, Yang Ying, Oi Litong. Peking University First Hospital

Objective To explore which factors influence PASP calculated by echocardigraph through tricuspid regurgitation pressure gradient.

Methods The retrospective study recruited 869 consecutive inpatients of Department of Cardiology in Peking University First Hospital, excluding patients who had acute myocardial infarction, pericardial effusion, congenital heart diseases, acute pulmonary embolism and organic tricuspid diseases. Their admission NYHA classification, N-terminal pro-B-type natriuretic peptide (NTproBNP), echocardiographic and other clinic data were collected. Pulmonary artery systolic pressure (PASP) was derived from transtricuspid regurgitation pressure gradient (TRPG) by echocardigraph. We analysed the correlations between PASP and age, sex, renal function, cardiopulmonary diseases and echocardigraph parameters, using single factor analysis and multivariate linear regression analysis.

Results Among these patients, 658 were found to have TR, with a proportion of $75.7 \%$. PASP was independently correlated to chronic obstructive pulmonary disease (COPD) (B (SE): 2.489 (1.121), $\mathrm{p}=0.027$ ), chronic pulmonary embolism (B (SE): 9.282 (2.175), $\mathrm{p}<0.001)$, aortic stenosis (B (SE): 13.846 (3.545), $\mathrm{p}<0.001$ ), aortic regurgitation ( $\mathrm{B}(\mathrm{SE}): 2.386$ (1.091), $\mathrm{p}=0.029)$, mitral regurgitation (B (SE): $2.093(0.934), p=0.025)$ and hypertension ( $\mathrm{B}(\mathrm{SE})$ : 1.560 (0.677), $\mathrm{p}=0.022)$, but not to other cardi- ovascular diseases such as cardiomyopathy, atrial fibrillation, coronary heart diseases and renal function $(p>0.05)$. PASP had independent correlation with both NYHA classification (B (SE): 3.701 (0.468), $\mathrm{p}=0.002$ ) and NT-proBNP (B (SE): 2.235 (0.569), $\mathrm{p}<0.001)$. PASP was correlated to TR severity positively (B (SE): 5.801 (0.798), $p<0.001)$, but not parallel to it. Age was an important predictor of PASP (B (SE): 0.081 (0.027), 95\% CI $(0.028$ to 0.134$), p<0.001)$, with an average increase in PASP of $0.81 \mathrm{~mm}$ $\mathrm{Hg}$ per decade.

Conclusions Tricuspid regurgitation is common in cardiac patients. PASP was independently correlated to COPD, chronic pulmonary embolism, left ventricular valve diseases and hypertension, but not to cardiomyopathy, atrial fibrillation, coronary heart diseases and renal function. PASP elevated with the increase of NYHA classification and NT-proBNP. It may play an important role in the evaluation of heart function. PASP should not be substituted by TR severity on the valuation of pulmonary circulation pressure. Age was an important predictor of PASP.

\section{e0665 CLINICAL FEATURE AND FOLLOW-UP RESULTS OF 60 PATIENTS WITH PULMONARY ARTERY HYPERTENSION}

doi:10.1136/hrt.2010.208967.665

Yuemin Sun, Guoyan Zhao, Zhenwen Yang. General Hospital of Tianjin Medical University

Objective To review the clinical feature of pulmonary artery hypertension $(\mathrm{PAH})$ and analyse the results of following up.

Methods Clinical feature of $60 \mathrm{PAH}$ patients who was diagnosed as PAH from Dec 2007 to Dec 2009 were reviewed retrospectively and those patients were followed up.

Results Six patients (three patients were CTD) died and six patients lost contact during following up. Those patients were divided into two groups based on pathogeny, Pulmonary Arterial Hypertension Associated With Connective Tissue Diseases (PAHCTD) group $(n=23)$ and the Pulmonary Arterial Hypertension Associated With Non-Connective Tissue Diseases PAH-NonCTD) group $(n=25)$. The former patient's condition obvious more critical than the latter and 20 patients using PAH targeted drug. In the course of follow-up, the condition had relieved somewhat in the two group patients, but the former still serious than the latter.

Conclusion Many complicated reasons can lead to PAH. The patients of Pulmonary Arterial Hypertension Associated With Connective Tissue Diseases had high morbidity and unfavourable prognosis. It is beneficial for PAH-CTD patient's administrated with targeted drug treatment and pay attention to follow-up visit.

\section{Related Subjects: Cerebrovascular Disease and Stroke}

\section{e0666 VASOPRESSIN AND EPINEPHRINE VERSUS EPINEPHRINE ALONE IN MANAGEMENT OF PATIENTS WITH OUT OF HOSPITAL CARDIAC ARREST A METAANALYSIS}

doi:10.1136/hrt.2010.208967.666

Jing Xiaoli, Wang Dongping, Li Hui, Xiong Yan, Zhan Hong, Li Xin. The First Affiliated Hospital of Sun Yat-sen University

Objective A combination of vasopressin and epinephrine may be more effective than epinephrine alone in cardiopulmonary resuscitation, but evidence is lacking to make a clinical recommendation. Our meta-analysis conducted to estimate the efficacy of vasopressin 